\title{
Revealing the Culprit behind the Pediatric Respiratory Infections: A Step-up Approach
}

\author{
Subramanya N Krishnamurthy ${ }^{1}$, Liz Mary Paul ${ }^{2}$
}

\begin{abstract}
Respiratory infections are caused by a vast number of pathogens including bacteria, viruses, and fungi. Many of these have nonspecific symptoms and do not have a final diagnosis in resource-limited settings-due to either economic reasons or lack of diagnostic facilities. A good understanding of the tests available would be of immense help, especially in critically ill patients in whom knowing the exact causative organism is mandatory to shift from empirical treatment to a more specific treatment plan. There are even rapid test formats which have good sensitivity for common respiratory infections encountered in outpatient set-up.

Keywords: BioFire, Bronchiolitis, Pediatric respiratory infection, Pharyngitis, Pneumonia.

Pediatric Infectious Disease (2019): 10.5005/jp-journals-10081-1218
\end{abstract}

\section{INTRODUCTION}

Respiratory infections are the most common among pediatric infectious diseases in tropical climates. Lower respiratory infections are estimated to cause approximately 4 million deaths annually worldwide and are one of the leading causes of death among children under 5 years old. ${ }^{1}$ The most frequent bacterial cause of pneumonia globally is Streptococcus pneumoniae and killed 393,000 children aged under 5 years in $2015 .^{2}$ Among viral respiratory infections which goes largely undiagnosed, respiratory syncytial virus (RSV) is the most common pathogen with 34 million episodes reported annually, and majority of the deaths caused by this virus occur in low- and middle-income countries. ${ }^{3}$ Influenza is yet another virus causing global pandemics and exhibiting genetic variations, making control measures increasingly difficult and resource intense. Considering the Indian scenario, we have lacunae in diagnostic testing because of a multitude of factors like cost concerns and lack of knowledge. Century-old diagnostic techniques like Gram stain and bacterial culture still remain the only investigations ordered in many areas of the country. The differentiation of viral and bacterial infections is often from blood routine rather than revealing the causative pathogen. To add on, there are many dilemmas in pediatric practice like overlapping clinical manifestations, difficulty in eliciting signs unlike adults, and handling parent and child psychological aspects.

In this review, we would like to suggest a "Step-up" approach to all pediatricians and general practitioners in the country. Step-up implies an additional step from the conventional investigations ordered for respiratory infections. They include tests as simple as rapid cards to multiplex polymerase chain reaction (PCR) like BioFire. This review highlights the common respiratory pathogens encountered in day-to-day outpatient practice.

Why step-up? To get a specific diagnosis, to avoid unnecessary antibiotic use, and hence control the super bugs and most importantly "A probable differential diagnosis becomes a diagnosis with evidence."
${ }^{1}$ Department of Pediatrics, Vaidehi Institute of Medical Sciences, Bengaluru, Karnataka, India

${ }^{2}$ Saroj Diagnostic Laboratory, IQRAA Hospital, Kozhikode, Kerala, India Corresponding Author: Subramanya N Krishnamurthy, Department of Pediatrics, Vaidehi Institute of Medical Sciences, Bengaluru, Karnataka, India, Phone: +91 9448207993, e-mail: subramanya.nk@gmail.com

How to cite this article: Krishnamurthy SN, Paul LM. Revealing the Culprit behind the Pediatric Respiratory Infections: A Step-up Approach. Pediatr Inf Dis 2019;1(4):142-144.

Source of support: Nil

Conflict of interest: None

\section{Group I $\beta$-Hemolytic Streptococcal Pharyngitis}

Clinical manifestations: Sore throat, fever, tender anterior cervical adenopathy, pharyngeal and tonsillar exudate, and palatal petechiae.

Differential diagnosis of pharyngitis/tonsillitis: Adenoviral pharyngitis, rhinovirus, Epstein-Barr virus (EBV), and bacterial pharyngitis caused by group I Streptococcus/Haemophilus influenzae (serotypes other than b, non-typable).

Conventional workup: Complete blood count (CBC) and throat swab culture.

Step-up: Rapid antigen test for Streptococcus by SD Bioline/Rapid VIDI test Strep A.

Rapid antigen test for Streptococcus is a point of care test with immunochromatography principle.

Sensitivity: $87 \%$, specificity: $95 \%$.

Cost of test: $\sim$ Rs 1,000/-.

Balasubramanian et al. have evaluated a rapid antigen detection test (RADT), and the sensitivity was $89.7 \%$ and specificity was $98.4 \%$ with a positive predictive value of $94.6 \%$, a negative predictive value of $96.9 \%$, and a diagnostic accuracy of $96.4 \% .{ }^{4}$

o The Author(s). 2019 Open Access This article is distributed under the terms of the Creative Commons Attribution 4.0 International License (https://creativecommons. org/licenses/by-nc/4.0/), which permits unrestricted use, distribution, and non-commercial reproduction in any medium, provided you give appropriate credit to the original author(s) and the source, provide a link to the Creative Commons license, and indicate if changes were made. The Creative Commons Public Domain Dedication waiver (http://creativecommons.org/publicdomain/zero/1.0/) applies to the data made available in this article, unless otherwise stated. 


\section{Epstein-Barr Viral Pharyngitis}

Clinical manifestations: Fever, pharyngitis, cervical lymphadenopathy, and hepatosplenomegaly.

Differential diagnosis of pharyngitis/tonsillitis: Adenoviral pharyngitis, rhinovirus, EBV, bacterial pharyngitis caused by group I Streptococcus/Haemophilus influenzae, and pharyngeal diphtheria. Conventional workup: $\mathrm{CBC} /$ peripheral smear and throat swab culture.

Step-up: EBV by enzyme immunoassay (EIA): IgM antibody to viral capsid antigen (VCA). Anti-VCA IgM appears early in EBV infection and usually disappears within 4 to 6 weeks.

Cost: Rs 1,500/-.

EBV DNA by PCR: Rs 3,000/-.

Luderer et al. have compared serological diagnosis of primary EBV infection and EBV reactivation with real-time EBV PCR. ${ }^{5}$ In a study by Bauer et al., EBV DNA detection exhibited a sensitivity of $94.9 \%$ and a specificity of $97.4 \%$ for primary EBV infection. ${ }^{6}$

\section{Community-acquired Pneumonia}

Clinical manifestations: High-grade fever, cough, and tachypnea. Differential diagnosis: Viral/bacterial/atypical pneumonia.

Conventional workup: CBC, C-reactive protein (CRP), chest X-ray, and blood culture.

Step-up: Urine pneumococcal antigen test, mycoplasma IgM, atypical pneumonia panel, and BioFire respiratory panel.

- Urine pneumococcal antigen test: Latex agglutination test; cost: $\sim$ Rs 1,200/-.

Mark and Marvin evaluated this test, and the high sensitivity was noted for proven (bacteremic) and suspected (focal pneumonia) invasive pneumococcal infections. However, false-positive test results were observed among febrile children without identified pneumococcal infection, approximately $15 \% .^{7}$

- Mycoplasma IgM/lgG: Method-chemiluminescence immunoassay; cost: Rs 2,000/-.

Qu et al. compared Mycoplasma pneumoniae detected by lgG and IgM antibody tests, fluorescence quantitative polymerase chain reaction (FQ-PCR), and culture. All the three methods exhibited high specificity values of around $90 \%$. Sensitivity was highest for culture (55.6\%) followed by FQ-PCR and serology. A similar trend was observed for positive predictive value and positive likelihood ratio. ${ }^{8}$

- Atypical pneumonia panel by ElAlgM antibodies to Legionella pneumophila; Mycoplasma pneumoniae; Coxiella burnetii; Chlamydia pneumoniae; adenovirus; RSV; influenza A/B; and parainfluenza 1,2 , and 3 .

Cost: Rs 6,500/-.

Dorairaj et al. described the use of enzyme-linked immunosorbent assay for diagnosing atypical pneumonia, whereas culture is difficult and requires special media and prolonged incubation. ${ }^{9}$

- Pneumoslide IgM, IFA

Indirect immunofluorescence assay in serum sample.

Detects Mycoplasma, Chlamydia, Legionella, Coxiella, RSV, influenza, adenovirus, parainfluenza.

\section{BRONCHIOLITIS-RSV}

Clinical manifestations: Upper respiratory tract symptoms such as fever, rhinorrhea, and congestion, followed by lower respiratory tract symptoms such as increasing cough, wheezing, and increased respiratory effort.
Differential diagnosis: Human rhinovirus, human metapneumovirus, influenza, adenovirus, coronavirus, and parainfluenza viruses.

Common investigations: $\mathrm{CBC}, \mathrm{CRP}, \mathrm{X}$-ray, and H1N1 PCR from throat swab/nasopharyngeal aspirate.

Step-up: RSV rapid antigen test/EIA for IgG or IgM antibodies, conventional multiplex PCR-respiratory panel, and BioFire respiratory panel.

- RSV rapid antigen test: Immunochromatography test:

SD Bioline RSV antigen test claims a sensitivity of $92.3 \%$ and specificity of $93.3 \%$. Chartrand et al. performed a systematic review and meta-analysis to evaluate the accuracy of RADTs for the diagnosis of RSV infection and reported sensitivity and specificity of 80 and $97 \%$, respectively. Higher sensitivity was seen in children compared with adults. ${ }^{10}$

- RSV IgG/lgM: EIA; cost: $\sim$ Rs 2,000/-.

- Conventional multiplex PCR:

Detects limited number of pathogens, relatively less specificity, lesser cost compared with BioFire.

Cost depends on the panel selected: $\sim$ Rs 3,000 to 8,000/-.

- BioFire FilmArray-Respiratory panel:

Nested multiplex PCR.

Elaborate panel including viruses and bacteria.

Adenovirus, coronavirus, human metapneumovirus, human rhinovirus/enterovirus, and influenza.

Middle East respiratory syncytial corona virus (Mers-CoV); influenza B; and parainfluenza 1, 2, 3, and 4.

RSV

Bordetella pertussis

Bordetella parapertussis

Chlamydophila pneumoniae

Mycoplasma pneumoniae

Cost: $\sim$ Rs 16,000/-

Leber et al. provided data from a multicenter evaluation comparing FilmArray respiratory panel 2 (RP2) with PCR and sequencing. The overall percent agreement between the FilmArray RP2 and the comparator testing was $99.2 \%$. The FilmArray RP2 also demonstrated a negative percent agreement of $\geq 93.8 \%$ for all analytes. ${ }^{11}$

\section{Pertussis}

Clinical manifestations: Low-grade fever and cough, cough paroxysms, and well between episodes.

Conventional labs: CBC, CRP; other-chest X-ray, nasopharyngeal aspirate; culture-high specificity, cumbersome, availability, and sensitivity issues.

Step-up: IgG antibodies by EIA, Rs 2,000/-; conventional PCR/ BioFire respiratory panel.

Lee et al. performed a clinical evaluation and validation for the laboratory methods used in the diagnosis of Bordetella pertussis infection. Specificity was $100 \%$ for culture and PCR; however, sensitivity was higher for PCR (90.6\%) compared with culture (60.4\%). Culture and PCR had higher sensitivity during the first 2 weeks of cough, whereas serology showed higher sensitivity after the second week. ${ }^{12}$

\section{References}

1. Pneumonia: The forgotten killer of children. Geneva, The United Nations Children's Fund (UNICEF)/World Health Organization 
(WHO), 2006, available from: http://www.who.int/maternal_child_ adolescent/ documents/9280640489/en/).

2. GBD. 2015 Mortality and causes of death Collaborators. global, regional, and national life expectancy, allcause mortality, and causespecifi c mortality for 249 causes of death, 1980-2015: a systematic analysis for the global burden of disease study 2015. Lancet 2016;388(10053):1459-1544. DOI: 10.1016/S0140-6736(16)31012-1.

3. Nair H, Nokes DJ, Gessner BD, et al. Global burden of acute lower respiratory infections due to respiratory syncytial virus in young children: a systematic review and meta-analysis. lancet. 2010;375(9725):1545-1555. DOI: 10.1016/S0140-6736(10)602061Available from: http://www.thelancet.com/journals/lancet/article/ PIIS0140-6736(10)60206-1/abstract).

4. Balasubramanian S, Amperayani S, Dhanalakshmi K, et al. Rapid antigen diagnostic testing for the diagnosis of group $A$ betahaemolytic streptococci pharyngitis. Natl Med J India 2018;31(1):8-10. DOI: 10.4103/0970-258X.243433.

5. Luderer R, KokM, Niesters HG, et al. Real-time Epstein-Barr virus PCR for the diagnosis of primary EBV infections and EBV reactivation. Mol Diagn 2005;9(4):195-200. DOI: 10.1007/bf03260091.

6. Bauer CC, Aberle S, Kraupp TP, et al. Serum Epstein-Barr virus DNA load in primary Epstein-Barr virus infection. J Med Virol 2005;75(1):54-58. DOI: 10.1002/jmv.20237.
7. Mark IN, Marvin B. Evaluation of a rapid urine antigen assay for the detection of invasive pneumococcal disease in children. Pediatrics 2003;112(6):1279-1282. DOI: 10.1542/peds.112.6.1279.

8. Qu J, Gu L, Wu J, et al. Accuracy of IgM antibody testing, FQ-PCR and culture in laboratory diagnosis of acute infection by mycoplasma pneumoniae in adults and adolescents with community-acquired pneumonia. BMC InfectDis 2013;13(1):172.DOI: 10.1186/1471-2334-13-172.

9. Dorairaj A, Kopula SS, Kumar K. Atypical pneumonia - screening in a tertiary care centre. J Clin Diagn Res 2015;9(11):DC18-DC20. DOI: 10.7860/JCDR/2015/16499.6835.

10. Chartrand C, Tremblay N, Renaud C, et al. Diagnostic accuracy of rapid antigen detection tests for respiratory syncytial virus infection: systematic review and meta-analysis. J Clin Microbiol 2015;53(12):3738-3749. DOI: 10.1128/JCM.01816-15.

11. Leber AL, Everhart K, Daly JA, et al. Multicenter evaluation of biofire FilmArray respiratory panel 2 for detection of viruses and bacteria in nasopharyngeal swab samples. J Clin Microbiol 2018;56(6):e01945-17. DOI: 10.1128/JCM.01945-17.

12. Lee AD, Cassiday PK, Pawloski LC, et al. Clinical evaluation and validation of laboratory methods for the diagnosis of Bordetella pertussis infection: culture, polymerase chain reaction (PCR) and antipertussis toxin IgG serology (IgG-PT). PLoS ONE 2018;13(4):e0195979. DOI: https://doi.org/10.1371/journal.pone.0195979. 